Pacific Journal of Mathematic 


\title{
ON ISOMETRIC ISOMORPHISM OF GROUP ALGEBRAS
}

\author{
J. G. WENDEL
}

1. Introduction. Let $G$ be a locally compact group with right invariant Haar measure $m[2$, Chapter XI $]$. The class $L(G)$ of integrable functions on $G$ forms a Banach algebra, with norm and product defined respectively by

$$
\begin{aligned}
\|x\| & =\int|x(g)| m(d g), \\
(x y)(g) & =\int x\left(g h^{-1}\right) y(h)_{m}(d h) .
\end{aligned}
$$

The algebra is called real or complex according as the functions $x(g)$ and the scalar multipliers take real or complex values.

Suppose that $\tau$ is an isomorphism (algebraic and homeomorphic) of the group $G$ onto a second locally compact group $\Gamma$ having right invariant Haar measure $\mu$; let $c$ be the constant value of the ratio $m(E) / \mu(\tau E)$, and let $\chi$ be a continuous character on $G$. If $T$ is the mapping of $L(G)$ onto $L(\Gamma)$ defined by

$$
(T x)(\tau g)=c \chi(g) x(g), \quad x \in L(G),
$$

then it is easily verified that $T$ is a linear map preserving products and norms; for short, $T$ is an isometric isomorphism of $L(G)$ onto $L(\Gamma)$.

It is the purpose of the present note to show that, conversely, any isometric isomorphism of $L(G)$ onto $L(\Gamma)$ has the above form, in both the real and complex cases.

We mention in passing that if $T$ is merely required to be a topological isomorphism then $G$ and $\Gamma$ need not even be algebraically isomorphic. In fact, let $G$ and $\Gamma$ be any two finite abelian groups each having $n$ elements, of which $k$ are of order 2. Then the complex group algebras of $G$ and $\Gamma$ are topologically isomorphic to the direct sum of $n$ complex fields, and the real algebras are topologically isomorphic to the direct sum of $k+1$ real fields and $(n-k-1) / 2$ two-dimensional algebras equivalent to the complex field. The algebraic content of this statement

Received October 24, 1950.

Pacific J. Math. 1 (1951), 305-311. 
follows from a theorem of Perlis and Walker [4], but for the sake of completeness we sketch a direct proof.

Since the character group of $G$ is isomorphic to $G$ there are exactly $k$ characters $\chi_{1}, \chi_{2}, \cdots, \chi_{k}$ on $G$ of order 2 . Together with the identity character $\chi_{0}$ these are all of the characters on $G$ which take only real values. The remaining characters $\chi_{k+1}, \cdots, \chi_{n-1}$ fall into complex-conjugate pairs, $\bar{X}_{2 m}=\chi_{2 m+1}, m=$ $(k+1) / 2,(k+3) / 2, \cdots,(n-2) / 2$. For $0 \leq j \leq n-1$ let $x_{j} \in L(G)$ (complex) be the vector with components $(1 / n) \chi_{j}(g)$. It is readily verified that the $x_{j}$ are orthogonal idempotents, so that $L(G)$ can be written as the sum of $n$ complex fields, and the same holds for the complex algebra $L(\Gamma)$. In the real case we retain the vectors $x_{j}$ for $0 \leq j \leq k$, and replace the remaining ones by the (real) vectors $y_{m}=x_{2 m}+x_{2 m+1}, z_{m}=i x_{2 m}-i x_{2 m+1}$, whose law of multiplication is easily seen to be $y_{m}^{2}=y_{m}, z_{m}^{2}=-y_{m}, y_{m} z_{m}=z_{m} y_{m}=z_{m}$, while all other products vanish. Since the vectors $x_{j}, y_{m}, z_{m}$ span $L(G)$ we see that $L(G)$ is represented as the sum of $k+1$ real fields and $(n-k-1) / 2$ complex fields; the same representation is obtained for the real algebra $L(\Gamma)$; this completes the proof of the algebraic part of the assertion. The fact that these algebras are also homeomorphic follows from the fact that all norms in a finite dimensional Banach space are equivalent.

2. Statement of results. For any fixed $g_{0} \in G$ let us denote the translation operator $x(g) \rightarrow x\left(g_{0}^{-1} g\right), x \in L(G)$, by $S_{g_{0}}$; operators $\Sigma_{\gamma}$ are defined similarly for $L(\Gamma)$. In this notation our precise result is:

THEOREM 1. Let $T$ be an isometric isomorphism of the (real, complex) algebra $L(G)$ onto the (real, complex) algebra $L(\Gamma)$. There is an isomorphism $\tau$ of $G$ onto $\Gamma$, and a (real, complex) continuous character $X$ on $G$ such that

$$
T S_{g} T^{-1}=\chi(g) \Sigma_{\tau g}
$$

where $c$ is the constant value of the ratio $m(E) / \mu(\tau E)$.

For the proof we make use of a theorem due to Kawada [3] concerning positive

\footnotetext{
*I am obliged to Professor C. E. Rickart for suggesting the probable existence of a formula of this kind.
} 
isomorphisms of $L(G)$ onto $L(\Gamma)$ in the real case; a mapping $P: L(G) \rightarrow L(\Gamma)$ is called positive in case $x(g) \geq 0$ a.e. in $G$ if and only if $(P x)(\gamma) \geq 0$ a.e. in $\Gamma$. Kawada's result reads:

THEOREM K. Let $P$ be a positive isomorphism of $L(G)$ onto $L(\Gamma)$, both algebras real. There is an isomorphism $\tau$ of $G$ onto $\Gamma$ such that $P S_{g} P^{-1}=k_{g} \Sigma_{\tau g}$, $g \in G$, where $k_{g}$ is positive for each $g$.

In order to deduce Theorem 1 from Theorem $\mathrm{K}$ we need two intermediate results, of which the first is a sharpening of Kawada's theorem, while the second reveals the close connection which holds between isometric and positive isomorphisms.

THEOREM 2. Let $P$ be a positive is omorphism of real $L(G)$ onto $L(\Gamma)$. Then:

$P$ is an isometry;

(2B) $k_{g}=1$ for all $g \in G$;

(2C) $P$ is given by the formula $(P x)(\tau g)=c x(g)$, where $c$ is the constant value of the ratio $m(E) / \mu(\tau E)$.

THEOREM 3. Let $T$ be an isometric isomorphism of $L(G)$ onto $L(\Gamma)$. There is a continuous character $\chi(\gamma)$ on $\Gamma$ such that if the mapping $P: L(G) \rightarrow L(\Gamma)$ is defined by $(P x)(\gamma)=\chi(\gamma)(T x)(\gamma), x \in L(G), \gamma \in \Gamma$, then $P$ is a positive is omorphism of the real subalgebra of $L(G)$ onto the real subalgebra of $L(\Gamma)$. The character $\chi$ is real or complex with $L(G)$ and $L(\Gamma)$.

3. Proof of Theorem 2. $P$ and its inverse are both order-preserving operators, and therefore are bounded $[1, \mathrm{p} .249]$. Consequently the ratio $\|P x\| /\|x\|$ is bounded away from zero and infinity as $x$ varies over $L(G), x \neq 0$. If $x$ is a positive element of $L(G)$ it follows by repeated application of Fubini's the orem that $\left\|x^{n}\right\|=\|x\|^{n}$; since $P x$ is also positive, and $P\left(x^{n}\right)=(P x)^{n}$, we have the result that for fixed positive $x \neq 0$ the quantity $\{\|P x\| /\|x\|\}^{n}$ is bounded above and below for $n=1,2, \cdots$. Hence $P$ is isometric at least for the positive elements of $L(G)$. But now for any $x \in L(G)$ we may write $x=x^{+}+x^{-}$, where $x^{+}$and $x^{-}$ denote respectively the positive and negative parts of $x$. Then

$$
\|x\|=\left\|x^{+}+x^{-}\right\|=\left\|x^{+}\right\|+\left\|x^{-}\right\|=\left\|P x^{+}\right\|+\left\|P_{x}^{-}\right\| \geq\left\|P x^{+}+P x^{-}\right\|=\|P x\| .
$$

Applying the argument to $P^{-1}$ we obtain the result

$$
\|x\|=\left\|P^{-1} P x\right\| \leq\|P x\| \leq\|x\| \text {, }
$$


which is the statement $(2 \mathrm{~A})$.

Theorem (2B) follows at once from this and Theorem K. For if $x \in L(G)$ then $\left\|S_{g} x\right\|=m_{g}\|x\|$, where $m_{g}$ is the constant value of the ratio $m(g E) / m(E)$. Similarly, $\left\|\Sigma_{\tau g} \xi\right\|=\mu_{\tau g}\|\xi\|$. Since $\tau$ is a homeomorphism, $\mu_{\tau g}=m_{g}$. The constant $k_{g}$ may now be evaluated by taking norms on both sides of the equation $P S_{g} P^{-1}$ $=k_{g} \Sigma_{\tau g}$, and must therefore have the value unity.

To prove part $(2 \mathrm{C})$ of the theorem we observe that the operator $Q$ defined by $(Q x)(\tau g)=c x(g)$ satisfies the relation $Q S_{g} Q^{-1}=\Sigma_{\tau g}$, and is an isomorphism of $L(G)$ onto $L(\Gamma)$. Then $Q S_{g} Q^{-1}=P S_{g} P^{-1}, g \in G$, and consequently $R=P^{-1} Q$ is a continuous automorphism of $L(G)$ which commutes with every $S_{g}$. We shall show that $R$ must be the identity mapping.

Segal $[5, \mathrm{p} .84]$ has shown that the product $x y$ of two elements $x, y$ belonging to $L(G)$ may be written as a Bochner integral, which in our notation takes the form

$$
x y=\int x(h) m_{h}^{-1}\left\{S_{h} y\right\} m(d h),
$$

where the quantity in braces is a vector-valued function of $h \in G$, and the function $m_{g}$ was defined above. Applying the operator $R$ we obtain

$$
R(x y)=\int x(h) m_{h}^{-1}\left\{R S_{h} y\right\} m(d h)=\int x(h) m_{h}^{-1}\left\{S_{h} R y\right\} m(d h)=x R y .
$$

But $R$ is an automorphism, and so also $R(x y)=(R x)(R y)$. Thus $x=R x$, all $x \in L(G)$, which shows that $P=Q$, as was to be proved.

4. Proof of Theorem 3. We first require several lemmas, all of which share the hypothesis: $T$ is an isometric isomorphism of $L(G)$ onto $L(\Gamma)$, indifferently real or complex. For $x, y \in L(G)$ we write $\xi$ for $T x, \eta$ for $T y$. We denote by $E(x)$ the set $\{g \mid g \in G, x(g) \neq 0\}$, which is regarded as being determined only up to a null-set; $E(\xi)$ in $\Gamma$ is defined in the same fashion. (Although we make no use of this fact, the first three lemmas below actually hold in case $T$ is an isometry between two arbitrary $L$-spaces.)

LemMA 1. If $E(x) \cap E(y)=\Lambda$ then $E(\xi) \cap E(\eta)=\Lambda$, and conversely.

Proof. The hypotheses imply that for all scalars $A$ we have $\|x+A y\|=\|x\|$ $+|A|\|y\|$. Then for all $A$ we have $\|\xi+A \eta\|=\|\xi\|+|A|\|\eta\|$, which implies that $E(\xi)$ and $E(\eta)$ are disjoint. For the converse we need only replace $T$ by $T^{-1}$. 
Lemma 2. If $E(x) \subseteq E(y)$ then $E(\xi) \subseteq E(\eta)$, and conversely.

Proof. Suppose that $E(x) \subseteq E(y)$, but that $E(\xi) \nsubseteq E(\eta)$. Then we may write $\xi=\xi_{1}+\xi_{2}$, with $E\left(\xi_{1}\right) \subseteq E(\eta), E\left(\xi_{2}\right) \cap E(\eta)=\Lambda=E\left(\xi_{1}\right) \cap E\left(\xi_{2}\right)$. Let $T^{-1} \xi_{i}=x_{i}$; then from Lemma 1 it follows that $E\left(x_{1}\right) \cap E\left(x_{2}\right)=\Lambda=E\left(x_{2}\right)$ $\cap E(y)$. But $E\left(x_{1}\right) \cup E\left(x_{2}\right)=E(x) \subseteq E(y)$; this contradiction yields the result.

Leмma 3. Let $B$ in $\Gamma$ be a $\sigma$-finite measurable set (that is, the sum of a countable number of sets of finite measure). Then there is a positive $x \in L(G)$ such that $E(\xi)=B$.

Proof. Let $\eta \in L(\Gamma)$ be chosen so that $E(\eta)=B$. Let $y=T^{-1} \eta$, and set $x(g)=|y(g)|, g \in G$. Then $x \in L(G), E(x)=E(y)$, and therefore from Lemma 2 it follows that $E(\xi)=B$.

Lemma 4. Let $x$ and $y$ be positive elements of $L(G)$. For $\gamma \in E(\xi)$ let $K_{\xi}(\gamma)=\xi(\gamma) /|\xi(\gamma)|$, and define $K_{\eta}(\gamma)$ in similar fashion. Then $K_{\xi}(\gamma)=$ $K_{\eta}(\gamma)$ almost everywhere on $E(\xi) \cap E(\eta)$.

Proof. Since $x$ and $y$ were taken to be positive we have $\|x+y\|=\|x\|+\|y\|$. Therefore $\|\xi+\eta\|=\|\xi\|+\|\eta\|$. Then $|\xi(\gamma)+\eta(\gamma)|=|\xi(\gamma)|+|\eta(\gamma)|$ a.e. in $\Gamma$. Hence, since the functions $K$ have modulus 1 ,

$$
\left|K_{\xi}(\gamma) K_{\eta}(\gamma)^{-1}\right| \xi(\gamma)|+| \eta(\gamma)||=|\xi(\gamma)|+|\eta(\gamma)|
$$

a.e. in $E(\xi) \cap E(\eta)$. But then $K_{\xi}(\gamma) K_{\eta}(\gamma)^{-1}=1$ a.e. on $E(\xi) \cap E(\eta)$, as was to be proved.

LEMMA 5. There is a unique continuous character $\chi$ on $\Gamma$ with the property that for all positive $x \in L(G)$ we have $\xi(\gamma)=\chi(\gamma)|\xi(\gamma)|$ a.e.; $\chi$ is real or complex with $L(G)$ and $L(\Gamma)$.

Proof. Let $\Gamma_{0}$ be the open-closed invariant subgroup of $\Gamma$ generated by a compact neighborhood of the identity. Since $\Gamma_{0}$ is $\sigma$-finite we may apply Lemma 3 to obtain a positive $x \in L(G)$ such that $E(\xi)=\Gamma_{0}$. Now $x \geq 0$ implies that $\left\|x^{2}\right\|=\|x\|^{2}$; then also $\left\|\xi^{2}\right\|=\|\xi\|^{2}$. The element $\xi^{2}$ is given by the formula

$$
\xi^{2}(\gamma)=\int_{\Gamma} \xi\left(\gamma \delta^{-1}\right) \xi(\delta) \cdot \mu(d \delta)=\int_{\Gamma_{0}} \xi\left(\gamma \delta^{-1}\right) \xi(\delta) \mu(d \delta) .
$$

Since $x^{2}$ is also positive we have from Lemma 4 that $K_{\xi^{2}}(\gamma)=K_{\xi}(\gamma)$ a.e. on $E\left(\xi^{2}\right) \cap E(\xi) \subseteq \Gamma_{0}=E(\xi)$. Writing simply $K(\gamma)$ for the common value, we see 
that the relation $\xi^{2}(\gamma)=K(\gamma)\left|\xi^{2}(\gamma)\right|$ therefore holds in $\Gamma_{0}$ even outside of $E\left(\xi^{2}\right)$. Then

$$
\begin{aligned}
\left|\xi^{2}(\gamma)\right| & =K(\gamma)^{-1} \int_{\Gamma_{0}} \xi\left(\gamma \delta^{-1}\right) \xi(\delta) \mu(d \delta) \\
& =\int_{\Gamma_{0}} K(\gamma)^{-1} K\left(\gamma \delta^{-1}\right) K(\delta)\left|\xi\left(\gamma \delta^{-1}\right) \xi(\delta)\right| \mu(d \delta) .
\end{aligned}
$$

Integrating over $\Gamma_{0}$ again we obtain

$$
\begin{aligned}
\left\|\xi^{2}\right\| & =\int_{\mu}^{-} \mu(d \gamma) \int K(\gamma)^{-1} K\left(\gamma \delta^{-1}\right) K(\delta)\left|\xi\left(\gamma \delta^{-1}\right) \xi(\delta)\right| \mu(d \delta) \\
& =\|\xi\|^{2}=\int \mu(d \gamma) \int\left|\xi\left(\gamma \delta^{-1}\right) \xi(\delta)\right| \mu(\dot{d} \delta) .
\end{aligned}
$$

Therefore $K(\gamma)^{-1} K\left(\gamma \delta^{-1}\right) K(\delta)=1$ a.e. on $\Gamma_{0} \times \Gamma_{0}$. Then there is a null-set $N \subset \Gamma_{0}$ such that $\gamma \notin N$ implies $K\left(\gamma \delta^{-1}\right) K(\delta)=K(\gamma)$ for almost all $\delta \in \Gamma_{0}$. We integrate this equation over a set $M$ of finite positive measure and obtain

$$
\begin{aligned}
K(\gamma) \mu(M) & =\int_{\Gamma_{0}} K\left(\gamma^{-1}\right) K(\delta) \phi_{M}(\delta) \mu(d \delta) \\
& =\int_{\Gamma_{0}} K\left(\delta^{-1}\right) K(\delta \gamma) \phi_{M}(\delta \gamma) \mu(d \delta),
\end{aligned}
$$

where $\phi_{M}$ is the characteristic function of $M$. The right member is easily seen to be a continuous function of $\gamma$, for all $\gamma \in \Gamma_{0}$; hence $K(\gamma)$ is equal a.e. to a continuous function $\chi_{0}(\gamma)$, which is clearly a character on $\Gamma_{0}$. From Lemma 4 it follows also that, for positive $x \in L(G)$, if $E(\xi) \subseteq \Gamma_{0}$ then $\xi(\gamma)=\chi_{0}(\gamma)$ $|\xi(\gamma)|$ a.e.

The proof is completed by extending the function $\chi_{0}$ to all of $\Gamma$. To do this we write $\Gamma$ as the union of disjoint cosets $\gamma_{\alpha} \Gamma_{0}$, and consider the open-closed subgroup $\Gamma_{1}$ generated by any finite number of cosets. Then $\Gamma_{1}$ is again $\sigma$-finite, and we may repeat the above argument to obtain a continuous character $\chi_{1}$ on $\Gamma_{1}$. Lemma 4 guarantees that for two such subgroups $\Gamma_{1}$ and $\Gamma_{1}^{\prime}$ the characters $\chi_{1}$ and $\chi_{1}^{\prime}$ will agree on $\Gamma_{1} \cap \Gamma_{1}^{\prime} \supseteq \Gamma_{0}$, so that $\chi_{1}$ is indeed an extension of $\chi_{0}$. Clearly, if $x \geq 0$ and $E(\xi) \subseteq \Gamma_{1}$ then $\xi(\gamma)=\chi_{1}(\gamma)|\xi(\gamma)|$.

Finally, $\chi$ on all of $\Gamma$ is defined by $\chi(\gamma)=\chi_{1}(y)$ for $y \in \Gamma_{1}$. Since the union of all such subgroups $\Gamma_{1}$ is precisely $\Gamma$, and since as shown above the subgroup 
characters are mutually consistent, the function $\chi$ is well-defined. It is clearly a continuous character. The remaining property, that $x \geq 0$ implies $\xi(\gamma)=\chi(\gamma)$ $|\xi(\gamma)|$, can be proved as follows. The set $E(\xi)$ intersects at most a countable number of cosets $\gamma_{n} \Gamma_{0}$ in sets of positive measure. Let $\xi_{n}$ be the restriction to $\gamma_{n} \Gamma_{0}$ of $\xi$, and put $x_{n}=T^{-1} \xi_{n}$. Then $x=\sum_{n=1}^{\infty} x_{n}$, and by Lemma 1 the sets $E\left(x_{n}\right)$ are pairwise disjoint, so that the $x_{n}$ are themselves positive elements. From this it follows that $\xi_{n}(\gamma)=\chi_{n}(\gamma)\left|\xi_{n}(\gamma)\right|=\chi(\gamma)\left|\xi_{n}(\gamma)\right|$ for $\gamma \in \gamma_{n} \Gamma_{0}$; hence the result holds.

The proof of Theorem 3 is now immediate. For the continuous character $\chi$ on $\Gamma$ constructed in Lemma 5 the mapping $P$ on $L(G)$ to $L(\Gamma)$ defined by $(P x)(\gamma)$ $=\chi(\gamma)^{-1}(T x)(\gamma)$ carries positive elements of $L(G)$ into positive elements of $L(\Gamma) ; P$ is clearly an algebraic isomorphism of $L(G)$ onto $L(\Gamma)$. We have only to show that $P x$ positive implies $x$ positive. Suppose then that $P_{x}=\xi$ is positive, but that $x=x_{1}-x_{2}+i\left(x_{3}-x_{4}\right)$, with $x_{j} \geq 0$ and $E\left(x_{1}\right) \cap E\left(x_{2}\right)=E\left(x_{3}\right) \cap E\left(x_{4}\right)$ $=\Lambda$, and correspondingly $\xi=\xi_{1}-\xi_{2}+i\left(\xi_{3}-\xi_{4}\right) . P$ is evidently an isometry, and therefore by Lemma 1 the sets $E\left(\xi_{1}\right) \cap E\left(\xi_{2}\right)$ and $E\left(\xi_{3}\right) \cap E\left(\xi_{4}\right)$ are null-sets. Therefore $\xi_{2}=\xi_{3}=\xi_{4}=0$; so $x=x_{1}$, and $x$ is positive.

5. Proof of Theorem 1. Because of Theorem 3 we may apply Theorems $\mathrm{K}$ and (2B) to the real sub-algebras of $L(G), L(\Gamma)$, to conclude that there is an isomorphism $\tau$ of $G$ onto $\Gamma$ such that $P S_{g} P^{-1}=\Sigma_{\tau g}$. Since $\tau$ is a homeomorphism we may regard the function $\chi$ as a continuous character on $G$, by defining $\chi(g)=$ $\chi(\tau g)$. By Theorem $(2 \mathrm{C}), P$ is given on the real subalgebras by the formula $(P x)$ $(\tau g)=c x(g)$, and, because of the linearity, this formula must hold throughout all of $L(G)$. Therefore $(T x)(\tau g)=c \chi(g) x(g)$, which proves (1B). Theorem (1A) is an easy consequence of this formula.

We note finally that Theorem (2A) shows that Kawada's theorem follows from Theorem 1 .

\section{REFERENGES}

1. Garrett Birkhoff, Lattice Theory, Amer. Math. Soc. Colloquium Publications, vol.25; American Mathematical Society, New York, 1948.

2. P. R. Halmos, Measure Theory, D. Van Nostrand, New York, 1949.

3. Y. Kawada, On the group ring of a topological group, Math. Japonicae 1 (1948), 1-5.

4. S. Perlis and G. L. Walker, Abelian group algebras of finite order, Trans. Amer. Math. Soc. 68 (1950), 420-426.

5. I. E. Segal, Irreducible representations of operator algebras, Bull. Amer. Math. Soc. 53 (1947), 73-88.

YALE UNIVERSITY 



\title{
PACIFIC JOURNAL OF MATHEMATICS
}

\section{EDITORS}

\author{
Herbert BuSEMANN \\ R. M. RoBINSON \\ University of Southern California \\ University of California \\ Los Angeles 7, California \\ Berkeley 4, California \\ E. F. BEC KENBACH, Managing Editor \\ University of California \\ Los Angeles 24, California
}

\section{ASSOCIATE EDITORS}
R. P. DILWORTH
P. R. HALMOS
BØRGE JESSEN
J. J. STOKER
HERBERT FEDERER
HEINZ HOPF
PAUL LÉVY
MARSHALL HALL
R. D. JAMES
GEORGE PÓLYA
E. G. STRAUS
KÖSAKU YOSIDA

\section{SPONSORS}

UNIVERSITY OF BRITISH COLUMBIA

CALIFORNIA INSTITUTE OF TECHNOLOGY

UNIVERSITY OF CALIFORNIA, BERKELEY

UNIVERSITY OF CALIFORNIA, DAVIS

UNIVERSITY OF CALIFORNIA, LOS ANGELES

UNIVERSITY OF CALIFORNIA, SANTA BARBARA

OREGON STATE COLLEGE

UNIVERSITY OF OREGON
UNIVERSITY OF SOUTHERN CALIFORNIA

STANFORD UNIVERSITY

WASHINGTON STATE COLLEGE

UNIVERSITY OF WASHINGTON

AMERICAN MATHEMATICAL SOCIETY

NATIONAL BUREAU OF STANDARDS, INSTITUTE FOR NUMERIGAL ANALYSIS

Mathematical papers intended for publication in the Pacific Journal of Mathematics should be typewritten (double spaced), and the author should keep a complete copy. Manuscripts may be sent to any of the editors. All other communications to the editors should be addressed to the managing editor, E. F. Beckenbach, at the address given above.

Authors are entitled to receive 100 free reprints of their published papers and may obtain additional copies at cost.

The Pacific Journal of Mathematics is published quarterly, in March, June, September, and December. The price per volume (4 numbers) is $\$ 8.00$; single issues, $\$ 2.50$. Spécial price to individual faculty members of supporting institutions and to members of the American Mathematical Society: $\$ 4.00$ per volume; single issues, $\$ 1.25$.

Subscriptions, orders for back numbers, and changes of address should be sent to the publishers, University of California Press, Berkeley 4, California.

UNIVERSITY OF CALIFORNIA PRESS - BERKELEY AND LOS ANGELES 


\section{Pacific Journal of Mathematics}

\section{Vol. 1, No. $2 \quad$ December, 1951}

Tom M. (Mike) Apostol, On the Lerch zeta function ................. 161

Ross A. Beaumont and Herbert S. Zuckerman, A characterization of the subgroups of the additive rationals ....................... 169

Richard Bellman and Theodore Edward Harris, Recurrence times for the Ehrenfest model................................... 179

Stephen P.L. Diliberto and Ernst Gabor Straus, On the approximation of a function of several variables by the sum of functions of fewer

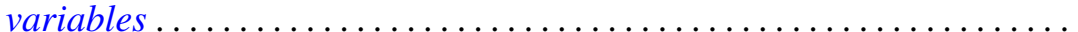

Isidore Isaac Hirschman, Jr. and D. V. Widder, Convolution transforms with complex kernels ................................ 211

Irving Kaplansky, A theorem on rings of operators .............. 227

W. Karush, An iterative method for finding characteristic vectors of a symmetric matrix............................... 233

Henry B. Mann, On the number of integers in the sum of two sets of positive integers ......................................... 249

William H. Mills, A theorem on the representation theory of Jordan

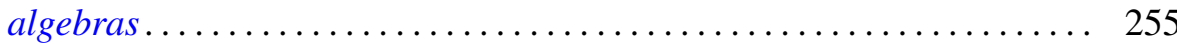

Tibor Radó, An approach to singular homology theory.............. 265

Otto Szász, On some trigonometric transforms ................... 291

James G. Wendel, On isometric isomorphism of group algebras ......... 305

George Milton Wing, On the $L^{p}$ theory of Hankel transforms ... 